\title{
The Acceleration of Outer-Belt Electrons by Localized Electric Fields
}

\author{
A. P. Kropotkin* \\ Skobeltsyn Institute of Nuclear Physics, Lomonosov Moscow State University, Moscow, 119234 Russia \\ *e-mail:apkrop@dec1.sinp.msu.ru \\ Received July 22, 2020; revised October 24, 2020; accepted January 28, 2021
}

\begin{abstract}
To explain the populations of the outer-belt energetic electrons, including relativistic electrons, that sporadically appear in the magnetosphere, a mechanism was proposed long ago for the acceleration of those electrons by short-term bursts of the electric field, which appear on the night side during substorm disturbances (Kropotkin, 1996). This mechanism can be substantially specified if the modern concepts of bursty bulk flows in the geomagnetic tail, the occurrence of dipolarization fronts, and the excitation of localized field-aligned-resonant poloidal Alfvén oscillations involving a strong component of the electric field in the dawn-dusk direction are taken into account.
\end{abstract}

DOI: $10.1134 /$ S0016793221030099

\section{INTRODUCTION}

It is known that relativistic electrons, which appear from time to time in the near-Earth part of the magnetosphere, are one of the most important factors of "space weather" (e.g., the modern review by Baker et al. (2018)). This effect has been discussed in the literature over the past decades, but a reliable, generally accepted theory has not been constructed to date. Here, we will continue our earlier research in this direction (Kropotkin, 1996). In doing so, we will rely on a number of important theoretical and experimental achievements of recent times.

The region of the outer electron radiation belt, where relativistic electrons are mainly observed, partially coincides on the night side and, as a rule, is slightly displaced to the Earth relative to the region in which the most intense magnetic-field variations occur during a substorm: these include alternating stretching of field lines and their dipolarization. This region has a relatively small radial extent, so that the $z$ component of the field there strongly decreases in the radial direction at a short distance (Lui, 1992; Kropotkin and Lui, 1995). This means that a small radial displacement of the near-equatorial particle, which occurs adiabatically (the first invariant $\mu=$ const), should be accompanied there by its significant acceleration.

It was determined long ago that such transport and acceleration should occur in this area during substorm activations, which occur with rapid dipolarization of the magnetic field and with intense transient electric fields directed from dawn to dusk (Lopez el al., 1989; Kropotkin, 1990). For the dominant plasma population there, with ion energies up to tens of $\mathrm{keV}$, this appears to be a substorm "injection" process (McIlwain and Whipple, 1986). The longitudinal drift peri- ods of these particles are on the order of several hours. They are strongly influenced by convective transport, so they should not return to the region of night-side acceleration during the same substorm. On the contrary, the most energetic electrons, with drift periods of the order of minutes, can return to this region many times during the same substorm and, thus, increase their energy by several times in successive dipolarization events, which can occur many times during one substorm.

By now, these general considerations can be presented in a more concrete manner. In what follows, we will briefly consider a number of the existing provisions derived from modern experimental and theoretical research. They characterize the mechanism of the emergence of fast localized plasma flows, their relationship to the processes of magnetic reconnection, and the emergence of "dipolarization fronts," as well as the excitation of strong magnetohydrodynamic (MHD) oscillations by these flows in the inner part of the magnetosphere with a dipole-like magnetic field. It is the electric fields of these oscillations that are capable of producing a significant acceleration of energetic electrons.

\section{PHYSICAL MECHANISMS}

In the plasma sheet of the geomagnetic tail, bursts of the electric field directed from dawn to dusk are associated with localized short-term plasma flows, which have been well studied in multisatellite experiments (Birn et al., 2011; Birn et al., 2017; Gabrielse et al., 2017; Liu et al., 2013, 2014; Lui, 2015; Nakamura et al., 2017; Runov et al., 2011; Sergeev et al., 2009; Wiltberger et al., 2015; Kropotkin, 2019). These include 
bursty bulk flows (BBFs) with a wider localization, as well as dipolarization flux bundles (DFBs) with a narrower localization, usually within the BBF.

Such localized short-term plasma flows arise as a result of bursts of magnetic reconnection in the geomagnetic tail, which form extremely thin current structures in the plasma sheet, anisotropic forced kinetic current sheets (FKCS) (Domrin and Kropotkin, 2007; Zelenyi et al., 2016; Kropotkin, 2014; Kropotkin and Domrin, 2009; Domrin and Kropotkin, 2004; Kropotkin and Domrin, 1996, 2009; Kropotkin et al., 1997; Nakamura et al., 2006; Zhou et al., 2009). In the central part of such a sheet, under the action of the reconnection electric field, plasma is transported along the sheet to the Earth at a rate close to the local Alfvén speed, i.e., a fast localized short-term plasma flow arises.

When approaching the Earth, there is a sharp deceleration of the flow at the front edge of the plasma sheet: the formation of the "dipolarization front" (Birn et al., 2011; Lui, 2015; Runov et al., 2011; Sergeev et al., 2009). Appearing, thus, in a very short time on the quasi-dipole flux tube in its near-equatorial part, the plasma flow particles here give up their momentum in this short time. Such a fast process looks like a "hit" on "strings", that is on quasi-dipole flux tubes in this region that occurs in their near-equatorial part.

On quasi-dipole field lines, there are specific eigenmodes of MHD oscillations that are field-aligned-resonant and small-scale in the direction transverse to the background magnetic field (Leonovich and Mazur, 1993). Their excitation by means of external driving was studied earlier (Leonovich and Mazur, 2016). These oscillations can be excited by the arrival of a dipolarization front. In the first approximation, the problem is solved as an initial problem with a nonzero initial pulse specified in the equatorial part of the flux tube, far from its ionospheric roots, where the MHD wave should be reflected. After such reflections, a field-aligned- resonant oscillatory disturbance arises, with a finite amplitude determined by intensity of the initial pulse.

Those field-aligned- resonant MHD oscillations involve a dawn-dusk electric field component that results in local acceleration of energetic electrons of the outer radiation belt, which is associated in time with magnetospheric substorms. This should lead to strong increases in the fluxes of such electrons.

\section{DETAILS AND DISCUSSION}

Jet flows arise in the kinetic description of processes in the geomagnetic tail, during FKCS generation. Note that they cannot appear in the ideal MHD with infinite conductivity. In the MHD approximation, transverse motions in the magnetosphere are possible only at Alfvénic, short times, and these are MHD waves, not convective motions, contrary to the interpretation of the simulation results given by Birn and Hesse (2014) and some other papers. For relatively slow, convective jet flows unrelated to FKCS generation, finite ionospheric conductivity is important: it determines the intensity of transverse currents in the magnetosphere, which are caused by the inertial drift of ions during flow deceleration and are connected to the ionosphere by the system of field-aligned currents. Here, it should also be borne in mind that the effect of conductivity cannot be correctly taken into account in numerical simulation due to the presence of artificial, numerical dissipation.

The "hit" on quasi-dipole flux tubes occurring in their near-equatorial part, which is associated with the arrival of the dipolarization front, generates, as noted, a wavelike MHD disturbance on these tubes. Such a disturbance arising in the near-Earth tail region, localized over the local time angle, should develop as fieldaligned-resonant Alfvén oscillations in the poloidal mode (Leonovich and Mazur, 1993; Leonovich and Mazur, 2016). This effect should be visible at relatively short Alfvénic times, $\tau \sim S / V_{\mathrm{A}}$, where $S$ is the length of the flux tube and $V_{A}$ is the average value of the Alfvén speed.

The magnetic field-aligned scale of the disturbance is much larger than the transverse one, which makes it possible to separate the field-aligned dependence in a certain way. It should be a solution of the one-dimensional Sturm-Liouville problem for standing Alfvén oscillations on a field line. A field line located at a given equatorial radial distance $L$ resonates at a certain frequency $\omega$.

The transverse structure of the oscillatory mode, however, presents a complex problem. The radial structure can be calculated if the azimuthal scale is small (azimuthal wavenumber $m \gg 1$ ). Let us briefly recall the relevant theory (Leonovich and Mazur, 1993).

Assuming that the transverse scales are small in comparison with the field-aligned ones and choosing an orthogonal coordinate set $\left(x^{1}, x^{2}, x^{3}\right)$, where $x^{1}=$ const on each magnetic shell, $x^{2}=\varphi$ is the longitudinal angle, and $x^{3}$ is counted from the equator along the magnetic field line, we can express the disturbance electric field $\mathbf{E}$ in terms of the scalar potential:

$$
E_{j}=-\partial \Phi / \partial x^{j}, j=1,2 .
$$

If $x^{3}$ is the distance $l$ measured along the field line from the equator, we get the following equation for $\Phi$ :

$$
\left(\nabla_{1} \hat{L}_{T} \nabla_{1}+\nabla_{2} \hat{L}_{P} \nabla_{2}\right) \Phi=0
$$

where $\nabla_{j}=\partial / \partial x^{j}$, and 


$$
\begin{aligned}
& \hat{L}_{T}=\hat{L}_{T}(\omega)=\frac{\partial}{\partial l} p \frac{\partial}{\partial l}+p \frac{\omega^{2}}{A^{2}}, \\
& \hat{L}_{P}=\hat{L}_{P}(\omega)=\frac{\partial}{\partial l} \frac{1}{p} \frac{\partial}{\partial l}+\frac{1}{p} \frac{\omega^{2}}{A^{2}}
\end{aligned}
$$

with $p=\left(g_{2} / g_{1}\right)^{1 / 2}$ depending on $l$ due to the curvature of the field $\mathbf{B}_{0} ; A$ designates the Alfvénic speed.

Seeking a solution with a small transverse scale, it is possible to present the azimuthal dependence in the form $\sim e^{i m \varphi}$ with $m \gg 1$, so that $\nabla_{2} \varphi=i m \varphi$, and the radial dependence may be presented in the quasi-classical form

$$
\Phi=H\left(x^{1}, l, \omega\right) \exp (i Q+i m \varphi)
$$

where $Q=\int^{x^{1}} k_{1}(s, \omega) d s$; for $H$ we obtain

$$
\hat{L} H=0,
$$

where

$$
\begin{aligned}
\hat{L}=\hat{L}\left(x^{1}, k_{1}, m, \omega\right) & \equiv k_{1}^{2} \hat{L}_{T}+k_{2}^{2} \hat{L}_{P}=\frac{\partial}{\partial l} q \frac{\partial}{\partial l}+q \frac{\omega^{2}}{A^{2}}, \\
q & =p k_{1}^{2}+p^{-1} m^{2} .
\end{aligned}
$$

Disregarding the ionospheric absorption, we obtain the boundary condition at the ionospheric level $l_{ \pm}$:

$$
\left.H\right|_{l_{ \pm}}=0 .
$$

For the given $x^{1}$ and $\omega$, the equations (2) and (3) determine the eigenvalue problem for the parameter $\kappa=k_{1} / \mathrm{m}$.

The eigenfunctions $H_{N}\left(x^{1}, l, \omega\right),(N=1,2, \ldots)$, correspond to the eigenvalues $\kappa_{N}\left(x^{1}, \omega\right)$, with $N$ half-waves on the given field line. Fixing the wave number $m$, we obtain the spectrum

$$
k_{1 N, m}=m \kappa_{N}\left(x^{1}, \omega\right),
$$

and the corresponding quasi-classical phase $Q$ has the form

$$
Q=Q_{N, m}\left(x^{1}, \omega\right)=m \int^{x^{1}} \kappa_{N}(s, \omega) d s .
$$

Thus, the solution of the longitudinal eigenvalue problem determines the dependence on the transverse coordinate $x^{1}$ in the leading order of the WentzelKramers-Brillouin (WKB) approximation.

For every $x^{1}$, there are two limiting cases, $\kappa=0$ and $\kappa=\infty$, which correspond to purely poloidal and purely toroidal modes. In the first case, we have an eigenvalue problem in the form

$$
\hat{L}_{P}(\omega) H=0,\left.H\right|_{l_{ \pm}}=0,
$$

with the solutions $\omega=\Omega_{N}^{P}\left(x^{1}\right)$ and $H=P_{N}\left(x^{1}, l\right)$. In the second case,

$$
\hat{L}_{T}(\omega) H=0,\left.\quad H\right|_{l_{ \pm}}=0
$$

with solutions $\omega=\Omega_{N}^{T}\left(x^{1}\right)$ and $H=T_{N}\left(x^{1}, l\right)$. The difference $\Delta \Omega_{N}=\Omega_{N}^{T}-\Omega_{N}^{P}$ is the polarization splitting of the spectrum and is determined by the curvature of the magnetic field $\mathbf{B}_{0}$. For a mode at a given frequency, there are two special resonant surfaces (RSs): the poloidal (PRS) on the magnetic shell $x^{1}=x_{P N}^{1}(\omega)$ and the toroidal (TRS) one at $x^{1}=x_{T N}^{1}(\omega)$. The transparency region of that mode is located between these two points, $x_{P N}^{1}$ and $x_{T N}^{1}$. In (Leonovich and Mazur, 1993; Leonovich and Mazur, 2016) an approximate solution for this transparency region is constructed.

It was shown that the mode structure for a given frequency $\omega$ is a traveling rather than a standing wave. From the PRS, it propagates towards higher $L$, where the TRS is located and where the wave is completely absorbed. In this process, the polarization of the disturbance changes from purely poloidal (the magnetic field vector lies in the meridional plane) to purely toroidal (the field vector is perpendicular to that plane). Analysis of this transformation process leads to an estimate of its characteristic time $\sim m / \omega$ (Leonovich and Mazur, 2016, p. 316). This time is the duration of the train of oscillations on a particular magnetic shell with the electric-field component directed azimuthally; they are capable of accelerating electrons on their drift orbit. It is seen that the number of oscillation cycles in the train is estimated by the azimuthal wave number $m$.

This effect can be manifested by $P i 2$ oscillation trains (e.g., Antonova et al., 2000). These oscillations are apparently also recorded in observations in the geomagnetic tail, and they are reproduced in MHD simulation (e.g., Figs. 6, 7 of Birn et al. (2011) and comments by Gabrielse et al. (2017, p. 5071-5072)). The extent to which these effects are important remains unclear from MHD simulation, since there is always an artificial, numerical damping of disturbances in it. On the other hand, Pi2 excitation by the BBFs that appear from the geomagnetic tail during a substorm has long been described in the literature (e.g., Kepke and Kivelson, 1999; Kepke et al., 2001). Of course, the further development of the disturbance in the inner magnetosphere is a complex MHD process, which can be interpreted in a certain approximation as the linear interaction of different modes (e.g., Keiling et al., 2001). Due to the notorious complexity of this picture, it has not yet been possible to cite observational evidence of the excitation of specifically poloidal Alfvén oscillations during dipolarization/BBF.

For the mechanism of the acceleration of relativistic electrons, it is important to assess how the oscillatory structure of the field $E$, which exists in the indicated field-aligned, resonant poloidal oscillations (and, accordingly, in the Pi2 train), affect them. Apparently, it is essential that the oscillation train is 
short and rapidly damping, so that the initial acceleration impulse strongly dominates. The first, largest half-wave in this decreasing train is always associated with arrival of the dipolarization front from the geomagnetic tail; thus, the azimuthal electric field in it has the required direction for accelerating electrons. The "damping" is actually due to the propagation and transformation of modes excited by an external source, which initially have a poloidal polarization but then, as already mentioned, quickly change to the toroidal one. A source of the instantaneous-impulse type ("impact") with a very wide (formally, infinite) spectrum excites Alfvén waves on all magnetic shells at once. A monochromatic wave is excited on a given magnetic shell $x^{1}$, for which this wave is poloidal. Accordingly, the frequency $\omega$ of the wave is equal to the poloidal frequency of this shell, $\Omega_{P N}\left(x^{1}\right)$, and the polarization of the wave is poloidal (the magnetic field oscillates in the radial direction along the $x^{1}$ coordinate). Each of the monochromatic waves then runs in the radial direction, i.e., to its toroidal surface, and the polarization of the wave gradually turns from poloidal to toroidal as this propagation progresses.

The theoretical analysis of the problem of disturbances in the near-Earth part of the geomagnetic tail, where the plasma parameter beta is not small, already has a long history that is reflected in many publications (in particular, the article by Mazur et al. (2014) and the book by Leonovich et al. Mazur (2016), which also contains many references to earlier works). These disturbances, which can be generally considered ballooning modes, are studied in their possible connection with the problem of substorm disruption of equilibrium in the global magnetospheric system. In this work, we focus on the fact that modern observations indicate the development during a substorm of a number of separate BBFs from the geomagnetic tail that are localized in local time. Their most important manifestation is the emergence of "dipolarization fronts." Each such front leads to the appearance of an azimuthal electric-field pulse on quasi-dipole field lines, where the beta parameter is sufficiently small. Thus, the application of the theory of Leonovich and Mazur for the corresponding magnetic flux tubes turns out to be justified.

Problems similar to ours were solved in a recent work (Gabrielse et al., 2017). Using an empirical analytical model of multiple DFBs wrapped in BBFs moving towards the Earth, the authors show how near-equatorial electrons can travel long distances and be accelerated by hundreds of $\mathrm{keV}$ via betatron acceleration. The model parameters are selected based on observations made on four THEMIS satellites; this imposes restrictions on the speed, location of the flows, and the magnitude of magnetic and electric fields in the DFB events. Sharp, localized peaks in a "dipolarized" magnetic field have such strong spatial gradients $\nabla B$ that energetic electrons make a gradient drift in closed trajectories around these peaks as the peaks move towards the Earth. Further, the idea of conservation of the third adiabatic invariant is used under conditions in which the field changes on time scales exceeding the electron drift period. An energetic electron rotates around a sharp peak in magnetic field along a closed trajectory encircling an area of approximately constant magnetic flux. As the magnetic field in the flux tube increases, the area encircled by the drift trajectory of the electron decreases, and the electron does not escape into the surrounding plasma sheet while continuing to gain energy due to betatron acceleration. When the DFB reaches the inner magnetosphere, where the background field is strong, electrons quickly gain access to the previously inaccessible drift shells that encompass the Earth. Thus, DFBs facilitate the transfer of energetic electrons over long distances along the geomagnetic tail and their acceleration, throwing them into the inner magnetosphere and increasing their energy by hundreds of $\mathrm{keV}$.

Such a mechanism can be considered an alternative or, possibly, additional mechanism to the one we have considered. However, it should be borne in mind that its implementation still requires the formation of BBFs that lead to the formation of DFBs. We point out that this should happen exactly during the formation of a reconnection layer, a thin anisotropic FKCS. Another important point is that, upon entering the inner magnetosphere, with its quasi-dipole field geometry, the electron finds itself in the zone of action of the MHD disturbances that are characteristic of this particular region, the field-aligned-resonant poloidal modes considered by us above; thus, the acceleration should take place precisely due to the electric fields of those modes.

\section{SIMPLE ESTIMATES AND CONCLUSIONS}

It is still impossible to construct a quantitative estimate of the disturbance amplitudes for the electric field of magnetic field-aligned-resonant, poloidal modes that accelerate energetic electrons: we have only a linear theory of the effect. It is also still difficult to distinguish exactly the manifestations of the fieldaligned-resonant poloidal modes in satellite observations. This, of course, results in significant uncertainty in the application of our results to the interpretation of observations. Many researchers believe that substorm injections deliver only preaccelerated electrons (seed electrons), which are then accelerated to relativistic energies by very low-frequency (VLF) emissions (a "chorus"). Whether this is always so, we cannot say yet. Apparently, both situations are possible, with additional acceleration by the chorus emissions and without it, depending on the intensity and frequency of the occurrence of substorm disturbances in the tail.

As for the acceleration of electrons to relativistic energies, it should be noted that it is often associated in the literature with the effect of Pc5 pulsations, 
which have periods on the same order as the drift period of such electrons motion around the Earth. This should lead to nonadiabatic changes in their fluxes, accompanied by their acceleration (e.g., Romanova and Pilipenko, 2008; Lam, 2017). However, unlike our theory, in that approach, nothing indicates an association with specific disturbances characteristic of substorms.

For a rough estimate of the possible effects in the particle fluxes, we only note that the typical electricfield intensity during a dipolarization is on the order of $E \sim 10 \mathrm{mV} / \mathrm{m}$ (for instance, (Lui, 2015)); the local time range for a disturbance is $\Delta \phi \sim 3-5 \mathrm{~h} \mathrm{LT}$, and the radial distance estimate is $R \sim 5-8 R_{E}$. The period of azimuthal drift is $\tau \sim 22 / L \varepsilon \sim 1-4$ min for electrons with energy $\varepsilon \sim 1-2 \mathrm{MeV}$. The duration of the field $E$ action on electron is then $\Delta \tau \sim \tau(\Delta \phi / 2 \pi) \sim 10-40 \mathrm{~s}$; the energy gain is $\Delta \varepsilon \sim 200-500 \mathrm{keV}$; the increase in the magnetic field on the electron drift orbit is $\Delta B / B \sim 10-$ $50 \%$ (it is kept in mind that $\varepsilon / B=$ const). The typical energy spectrum at high energies in the outer radiation belt may be presented in the exponential form $J_{e} \sim \exp \left(-\varepsilon / \varepsilon_{0}\right)$ with $\varepsilon_{0} \sim 0.3 \mathrm{MeV}$. Based on these estimates, we obtain a flux increase at every acceleration act on the order of $\Delta J_{e} \sim 100-300 \%$.

Note that the estimate used above for the duration of the field $E$ action on electron is based on the assumption that this duration is determined simply by the duration of the stay of the drifting electron in the azimuthal sector of the disturbance. This means that such a time interval should be short as compared to the characteristic period of the disturbance. If the disturbance is associated, as we have done above, with the first half-wave of the decaying Pi2 pulsation train (their period is $\tau \sim 10^{2} \mathrm{~s}$ ), then the given estimate of $10-40 \mathrm{~s}$ makes sense. In general, the uncertainty in the observational data apparently does not yet allow us to go further.

Thus, we find confirmation of the previously made assumption (Antonova et al., 1999) that short, strong bursts of the electric field associated with local bursts of dipolarization, which, in turn, are short-duration manifestations of substorm activity on the night side, can strongly accelerate electrons with an initial energy in the range of $\sim 100 \mathrm{keV}$ to $\sim 1 \mathrm{MeV}$. This is a unidirectional process; thus, a series of successive impacts leads to ever greater energy gains. This process is regular rather than stochastic.

\section{OPEN ACCESS}

This article is licensed under a Creative Commons Attribution 4.0 International License, which permits use, sharing, adaptation, distribution and reproduction in any medium or format, as long as you give appropriate credit to the original author(s) and the source, provide a link to the
Creative Commons licence, and indicate if changes were made. The images or other third party material in this article are included in the article's Creative Commons licence, unless indicated otherwise in a credit line to the material. If material is not included in the article's Creative Commons licence and your intended use is not permitted by statutory regulation or exceeds the permitted use, you will need to obtain permission directly from the copyright holder. To view a copy of this licence, visit http://creativecommons.org/licenses/by/4.0/.

\section{REFERENCES}

Antonova, A.E., Gubar', Yu.I., and Kropotkin, A.P., Towards the model of relativistic electron fluxes: Acceleration in the field of strong Alfvénic disturbances, Radiat. Meas., 1999, vol. 30, pp. 515-521.

Antonova, A.E., Gubar', Yu.I., and Kropotkin, A.P., A model of spatio-temporal structure of the substorm electromagnetic disturbance and its consequences, Phys. Chem. Earth, 2000, vol. 25, nos. 1-2, pp. 43-46.

Baker, D.N., Erickson, P.J., Fennell, J.F., Foster, J.C., Jaynes, A.N., and Verronen, P.T., Space weather effects in the Earth's radiation belts, Space Sci. Rev., 2018, vol. 214, id 17. https://doi.org/10.1007/s11214-017-0452-7

Birn, J. and Hesse, M., The substorm current wedge: further insights from MHD simulations, J. Geophys. Res.: Space Phys., 2014, vol. 119, pp. 3503-3513. https://doi.org/10.1002/2014JA019863

Birn, J., Nakamura, R., Panov, E.V., and Hesse, M., Bursty bulk flows and dipolarization in MHD simulations of magnetotail reconnection, J. Geophys. Res., 2011, vol. 116, A01210. https://doi.org/10.1029/2010JA016083

Birn, J., Runov, A., and Zhou, X.Z., Ion velocity distributions in dipolarization events: Distributions in the central plasma sheet, J. Geophys. Res., 2017, vol. 122, pp. 8014-8025.

Domrin, V.I. and Kropotkin, A.P., Forced current sheet structure, formation and evolution: Application to magnetic reconnection in the magnetosphere, Ann. Geophys., 2004, vol. 22, pp. 2547-2553. https://doi.org/10.5194/angeo-22-2547-2004

Domrin, V.I. and Kropotkin, A.P., Dynamics of equilibrium upset and electromagnetic energy transformation in the geomagnetotail: A theory and simulation using particles. 3. Versions of formation of thin current sheets, Geomagn. Aeron. (Engl. Transl.), 2007, vol. 47, no. 5, pp. 555-565.

Gabrielse, C., Angelopoulos, V., Harris, C., Artemyev, A., Kepko, L., and Runov, A., Extensive electron transport and energization via multiple, localized dipolarizing flux bundles, J. Geophys. Res.: Space Phys., 2017, vol. 122, pp. 5059-5076. https://doi.org/10.1002/2017JA023981

Keiling, A., Wygant, J.R., Cattell, C., et al., Pi2 pulsations observed with the Polar satellite and ground stations: Coupling of trapped and propagating fast mode waves to a midlatitude field line resonance, J. Geophys. Res., 2001, vol. 106, pp. 25891-25904.

Kepko, E.L. and Kivelson, M.G., Generation of Pi2 pulsations by bursty bulk flows, J. Geophys. Res., 1999, 
vol. 104, pp. 25021-25034.

https://doi.org/10.1029/1999JA900361

Kepko, E.L., Kivelson, M.G., and Yumoto, K., Flow bursts, braking, and Pi2 pulsations, J. Geophys. Res., 2001, vol. 106, no. A2, pp. 1903-1915.

https://doi.org/10.1029/2000JA000158

Kropotkin, A.P., Dynamics of the geomagnetic tail plasma sheet and the magnetospheric substorm, Res. Geomagn. Aeron. Sol. Phys., 1990, vol. 89, pp. 119-133.

Kropotkin, A.P., Relativistic electron transport processes associated with magnetospheric substorms, Radiat. Meas., 1996, vol. 26, no. 3, pp. 343-345.

Kropotkin, A.P., Energy conversion and magnetic reconnection in space plasmas: Role of nonlinear kinetic processes and structures, Geomagn. Aeron. (Engl. Transl.), 2014, vol. 54, no. 4, pp. 397-405.

Kropotkin, A.P., Dipolarization flux bundles, Geomagn. Aeron. (Engl. Transl.), 2019, vol. 59, no. 2, pp. 162-169.

Kropotkin, A.P. and Domrin, V.I., Theory of a thin one-dimensional current sheet in collisionless space plasma, J. Geophys. Res., 1996, vol. 101, pp. 19893-19902.

Kropotkin, A.P. and Domrin, V.I., Geomagnetotail dynamics: Different types of equilibriums and transitions between them, Geomagn. Aeron. (Engl. Transl.), 2009a, vol. 49, no. 2, pp. 169-178.

Kropotkin, A.P. and Domrin, V.I., Kinetic thin current sheets: their formation in relation to magnetotail mesoscale turbulent dynamics, Ann. Geophys., 2009b, vol. 27 , no. 7 , pp. 1353-1362.

Kropotkin, A.P. and Lui, A.T.Y., Quasi-static evolution of the magnetosphere: The substorm growth phase, J. Geophys. Res., 1995, vol. 100, pp. 17231-17238.

Kropotkin, A.P., Malova, H.V., and Sitnov, M.I., The selfconsistent structure of a thin anisotropic current sheet, J. Geophys. Res., 1997, vol. 102, pp. 22099-22106.

Lam, H.-L., On the predictive potential of Pc5 ULF waves to forecast relativistic electrons based on their relationships over two solar cycles, Space Weather, 2017, vol. 15, pp. 163-179.

https://doi.org/10.1002/2016SW001492

Leonovich, A.S. and Mazur, V.A., A theory of transverse small-scale standing Alfvén waves in an axially symmetric magnetosphere, Planet. Space Sci., 1993, vol. 41, no. 9 , pp. $697-717$.

Leonovich, A.S. and Mazur, V.A., Lineinaya teoriya MGDkolebanii magnitosfery (Linear Theory of Magnetospheric MHD Oscillations), Moscow: Fizmatlit, 2016.

Liu, J., Angelopoulos, V., Runov, A., and Zhou, X.-Z., On the current sheets surrounding dipolarizing flux bundles in the magnetotail: The case for wedgelets, J. Geophys. Res.: Space Phys., 2013, vol. 118, pp. 2000-2020. https://doi.org/10.1022/jgra.50092

Liu, J., Angelopoulos, V., Zhou, X.-Z., and Runov, A., Magnetic flux transport by dipolarizing flux bundles, $J$. Geophys. Res.: Space Phys., 2014, vol. 119, pp. 909-926. https://doi.org/10.1002/2013JA019395
Lopez, R.E., Lui, A.T.Y., Sibeck, D.G., et al., On the relationship between the energetic particle flux morphology and the change in the magnetic field magnitude during substorms, J. Geophys. Res., 1989, vol. 94, pp. 17105-17119.

Lui, A.T.Y., Radial profiles of quiet time magnetospheric parameters, J. Geophys. Res., 1992, vol. 97, pp. 1932519332.

Lui, A.T.Y., Dipolarization fronts and magnetic flux transport, Geosci. Lett., 2015, vol. 2, id 15. https://doi.org/10.1186/s40562-015-0032-1

Mazur, N.G., Fedorov, E.N., and Pilipenko, V.A., Longitudinal structure of ballooning MHD disturbances in a model magnetosphere, Cosmic Res., 2014, vol. 52, no. 3 , pp. $175-184$.

Mcllwain C.E. and Whipple, E.C., The dynamic behaviour of plasmas near geosynchronous orbit, IEEE Trans. on Plasma Sci. PS-, 1986, pp. 874-890.

Nakamura, R., Baumjohann, W., Runov, A., and Asano, Y., Thin current sheets in the magnetotail observed by CLUSTER, Space Sci. Rev., 2006, vol. 122, nos. 1-4, pp. $29-38$.

Nakamura, R., Nagai, T., Birn, J., et al., Near-Earth plasma sheet boundary dynamics during substorm dipolarization, Earth Planets Space, 2017, vol. 69, pp. 129-142. https://doi.org/10.1186/s40623-017-0707-2

Romanova, N. and Pilipenko, V., ULF wave indices to characterize the solar wind-magnetosphere interaction and relativistic electron dynamics, Acta Geophys., 2008, vol. 57 , no. 1 , pp. $158-170$. https://doi.org/10.2478/s11600-008-0064-4

Runov, A., Angelopoulos, V., Zhou, X.-Z., Zhang, X.J., Li, S., Plaschke, F., and Bonnell, J., A THEMIS multicase study of dipolarization fronts in the magnetotail plasma sheet, J. Geophys. Res., 2011, vol. 116, A05216. https://doi.org/10.1029/2010JA016316

Sergeev, V.A., Angelopoulos, V., Apatenkov, S., Bonnell, J., Ergun, R., Nakamura, R., McFadden, J.P., Larson, D., and Runov, A., Kinetic structure of the sharp injection/dipolarization front in the flow braking region, Geophys. Res. Lett., 2009, vol. 36, L21105. https://doi.org/10.1029/2009GL040658

Wiltberger, M., Merkin, V., Lyon, J.G., and Ohtani, S., High-resolution global magnetohydrodynamic simulation of bursty bulk flows, J. Geophys. Res.: Space Phys., 2015, vol. 120, pp. 4555-4566. https://doi.org/10.1002/2015JA021080

Zelenyi, L.M., Malova, H.V., Grigorenko, E.E., and Popov, V.Yu., Thin current sheets: From the work of Ginzburg and Syrovatskii to the present day, Phys.Usp., 2016, vol. 59, no. 11, pp. 1057-1090.

Zhou, X.-Z., Angelopoulos, V., Runov, A., et al., Thin current sheet in the substorm late growth phase: Modeling of THEMIS observations, J. Geophys. Res.: Space Phys., 2009, vol. 114, A03223.

https://doi.org/10.1029/2008JA013777 